\title{
Notes on "Roux en Y Gastric Bypass Increases Ethanol Intake in the Rat" by Davis et al.
}

\author{
Andras Hajnal • Panayotis K. Thanos • Nora D. Volkow
}

Published online: 7 May 2013

(C) Springer Science+Business Media New York 2013

We read with great interest the article "Roux en $\mathrm{Y}$ Gastric Bypass Increases Ethanol Intake in the Rat" [1] from Jon Davis and colleagues, published online in the Obesity Surgery on February 26, 2013. This study reports an impressive data set showing increased alcohol consumption in an outbred rat strain after Roux-en-Y gastric bypass surgery (RYGB), a finding that has potential important clinical implications. This study supports recent clinical investigations showing increased susceptibility to alcohol abuse following RYGB [2-5] and corroborates our recent studies that reported increased alcohol taking and increased motivation to work for alcohol reward in dietary obese rats that received RYGB $[6,7]$.

However, we were surprised that the authors did not refer to prior publications showing similar outcomes [6, 7], which would have strengthen the conclusion of their paper. The authors also failed to cite their prior paper [8], which reported decreases in alcohol intake after RYGB in alcohol-preferring rats as well as decreased alcohol intake after RYGB in patients who regularly drank alcohol prior to undergoing surgery. The differences between their

\footnotetext{
A. Hajnal $(\bowtie)$

Department of Neural and Behavioral Sciences,

College of Medicine, The Pennsylvania State University, 500 University Drive, Mail code: H181, Hershey, PA 17033, USA e-mail: ahajnal@psu.edu
}

\section{A. Hajnal \\ Department of Surgery, The Pennsylvania State University College of Medicine, Hershey, PA, USA}

P. K. Thanos $\cdot$ N. D. Volkow

Laboratory of Neuroimaging, NIAAA Intramural Program,

$\mathrm{NIH}$, Bethesda, MD, USA

\section{P. K. Thanos}

Behavioral Neuropharmacology and Neuroimaging Lab, Brookhaven National Laboratory, Upton, NY, USA current results and their prior findings suggest that the effects of RYGB may be modulated by genetic background and should have been discussed rather than ignored.

There are increasing concerns over the lack of reproducibility of findings from preclinical research [9], yet the study of Davis et al. show similar findings to the ones we previously reported using a similar animal model and surgical procedure but done by an independent laboratory.

Conflict of Interest The authors have no conflict of interest to report.

\section{References}

1. Davis JF, et al. Roux en Y gastric bypass increases ethanol intake in the rat. Obes Surg. 2013. doi:10.1007/s11695-013-0884-4

2. Ertelt TW et al. Alcohol abuse and dependence before and after bariatric surgery: a review of the literature and report of a new data set. Surg Obes Relat Dis. 2008;4(5):647-50.

3. Hsu LK et al. Nonsurgical factors that influence the outcome of bariatric surgery: a review. Psychosom Med. 1998;60(3):338-46.

4. Saules KK et al. Bariatric surgery history among substance abuse treatment patients: prevalence and associated features. Surg Obes Relat Dis. 2010;6(6):615-21.

5. King WC et al. Prevalence of alcohol use disorders before and after bariatric surgery. JAMA. 2012;307(23):2516-25.

6. Hajnal A et al. Alcohol reward is increased after Roux-en-Y gastric bypass in dietary obese rats with differential effects following ghrelin antagonism. PLoS One. 2012;7(11):e49121.

7. Thanos PK et al. Gastric bypass increases ethanol and water consumption in diet-induced obese rats. Obes Surg. 2012;22(12): 1884-92.

8. Davis JF et al. Gastric bypass surgery attenuates ethanol consumption in ethanol-preferring rats. Biol Psychiatry. 2012;72(5):354-60.

9. Prinz F, Schlange T, Asadullah K. Believe it or not: how much can we rely on published data on potential drug targets? Nat Rev Drug Discov. 2011;10(9):712. 